\title{
Neural Networks for Modal and Virtual Learning
}

\section{Dominic Palmer-Brown}

\author{
Dean of Computing Faculty \\ London Metropolitan University \\ 166-220 Holoway Road, \\ London N7 8DB \\ UK, d.palmer-brown@londonmet.ac.uk
}

\begin{abstract}
This talk will explore the integration of learning modes into a single neural network structure in order to overcome the inherent limitations of any given mode (for example some modes memorize specific features, others average across features and both approaches may be relevant according to the circumstances). Inspiration comes from neuroscience, cognitive science and human learning, where it is impossible to build a serious model of learning without consideration of multiple modes; and motivation also comes from non-stationary input data, or time variant learning oblectives, where the optimal mode is a function of time. Several modal learning ideas will be presented, including the Snap-Drift Neural Network which toggles its learning (across the network or on a neuron-by-neuron basis) between two modes, either unsupervised or guided by performance feedback (reinforcement) and an adaptive function Neural Network (ADFUNN) in which adaption applies simultaneously to both the weights and the individual neuron activation functions. The talk will also focus on a virtual learning environment example that involves the modal learning Neural Network, identifying patterns of student learning that can be used to target diagnostic feedback that guides the learner towards increased states of knowledge.
\end{abstract}

Please use the following format when citing this chapter:

Palmer-Brown, D., 2009, in IFIP International Federation for Information Processing, Volume 296; Artificial Intelligence Applications and Innovations III; Eds. Iliadis, L., Vlahavas, I., Bramer, M.; (Boston: Springer), pp. 2-2. 